\title{
Agenda Control in Coalition Formation
}

\author{
Francis Blochy and Stéphane Rottier ${ }^{z}$
}

December 17, 1999

\begin{abstract}
A bstract
Theoretical models of government formation in political science usually assume that the head of state is non-strategic. In this paper, we analyze the power of an agenda setter who chooses the order in which players are recognized to form coalitions in simple games. We characterize those sets of players which can be imposed in the equilibrium coalition and show that the only decisive structures where the agenda setter can impose the presence of any minimal winning coalition are apex games, where a large player forms a winning coalition with any of the small players.
\end{abstract}

\footnotetext{
${ }^{x}$ We thank (without implicating) Michel Le Breton for his comments and Francesco de Sinopoli for his careful reading of the paper. Wearealso grateful to seminar participants in GREQAM, Ghent, CentER, DELTA and Cergy-Pontoise for helpful comments. Financial support from the CommunautéFrançaise deBelgique (programmed'Actions deRecherches Concertées 1999/04-235) is gratefully acknowledged

yIRES, Department of Economics, and CORE, Université catholique de Louvain, Belgium.

${ }^{z}$ RRES, Department of Economics, Université catholique de Louvain, Belgium.
} 


\section{Introduction}

Can a head of state choose the composition of a cabinet by selecting the order in which party leaders attempt to form the government? Can the chairman of a board decide on the composition of an executive committee by choosing the order in which board members speak? It is generally believed that the answer to these questions is at least partially positive: under some circumstances, the head of state or the chairman of the board can use their agenda setting power to inłuencethecoalitions formed as theoutcome of bargaining between parties or board members. In the political realm, the head of state's ability to infuence the formation of government coalitions varies from country to country. Whilevery few constitutions have speci..c provisions on the order in which party leaders are recognized to form governments, in some countries, the power of the head of state is limited by conventions, which dictate that the largest party should be asked to form the government .r.st. In other countries, like Finland or Italy, the President of the Republic has used his power to choose the order in which parties attempt to form the government (Strom, Budge and Laver, 1994).

In this paper, we develop a formal model of agenda control to analyze the power of the head of state or the chairman of the board in infuencing the coalitions formed. In particular, we relate the power of the agenda setter to the underlying decisive structure among players. Is it easier for a head of state to manipulate the outcome of coalitional bargaining when all parties have equal size, or when parties are asymmetric? Are small or large parties more likely to be imposed in the cabinet? How should the head of state design the order of moves in relation to the parties' weights? We provide an answer to these questions in the general context of cooperative simple games, where coalitions of players are divided between losing and winning coalitions.

More speci..cally, we suppose that the agenda setter selects an ordering 
of the players, and that bargaining occurs according to the following simple sequential procedure. When a player is recognized to make a proposal, she oxers to form a coalition with other players. If all coalition members agree, the coalition is formed and the game ends. If at least one of the players rejects the oxer, the next player in the sequence is recognized to make an oxer. If at the end of the sequence, no coalition has been formed, all players obtain their ..xed status quo outcome.

Our main result shows that the only structure in which the agenda setter can fully manipulate the outcome of coalitional bargaining is the apex structure, with one large player and $n$ i 1 small players, where the large player only needs one of the small players to form a winning coalition. In all other structures, the power of the agenda setter is limited: he may be able to force the presence of some of the players in the coalition formed, but cannot manipulate the order to guarantee that any possible minimal winning coalition is formed. If all players are symmetric, the agenda setter's power is even more limited: he can only choose one player to be included in the coalition, and one player to be excluded from the coalition.

Furthermore, for any simple game, we completely characterize the sets of players who can beincluded in the coalition formed by theagenda setter. We also show that when players can be ordered by desirability, as in parliaments, where parties are more desirable if they have more seats, the agenda setter should choose the least desirable coalition member to be the ..rst mover, and the most desirable member outside the coalition to be the second mover. In the context of cabinet formation, this implies that the head of state should recognize .r.st the smallest party he wants to include in the coalition, and let the largest party he wants to exclude be the second mover.

In the political science literature, the agenda setting role of the head of state is usually left unmodeled. For example, in their recent wideranging analysis of cabinet formation based on policy portfolios, Laver and Shepsle 
(1994, pp. 53-54) indicate that "It is at well to be quite dear that [they] assume that the recognition of the actors who can propose new governments is non-strategic. This implies for example that the head of state is not a strategic actor in thegovernment formation process". Similarly, Baron (1991, p. 137) notes that, in his noncooperative mode of government formation, "the political bargaining begins with a non-strategic president or monarch who selects a party to be accorded the opportunity to attempt to form a government". Hence, our paper contributes to the noncooperative theory of coalition formation in political science by endogenizing the agenda setting role of the head of state

Once the head of state has chosen the ordering of proposers, the model of coalitional bargaining we adopt is directly inspired by the noncooperative models of coalition formation initiated by Baron and Ferejohn (1989) and Austen-Smith and Banks (1988). As in Baron and Ferejohn (1989), we assume a purely redistributive framework, and do not incorporate an ide ological dimension in party preferences. (Se also Baron (1991 and 1993) and J ackson and Moselle (1998) for models of coalition formation where parties have preferences over policy outcomes.) In all these models, the order in which players are recognized is exogenously .xed. For example, Baron and Ferejohn (1989) assume that the probability of being recognized is proportional to the number of seats whereas Austen-Smith and Banks (1988) suppose that parties are given the opportunity to form a government in the order given by their number of seats. In recent empirical work, Merlo (1997) and Diermeier and Merlo (1999) investigatetheempirical validity of dixerent bargaining procedures. In particular, Diermeier and Merlo (1999) note that the data do not support the procedure described by Austen-Smith and Banks (1988), but give some validity to the proportional procedure of Baron and Ferejohn (1989). They also note the incumbent is more likely to be selected to attempt to form the new government. 
In the gametheoretic literature on coalitional bargaining, Chatterjee et al. (1993), Bloch (1996) and Ray and Vohra (1999) have analyzed sequential models where the order of proposers and responders is ..xed by an exoge nous protocol. These studies emphasize the role played by the protocol in the determination of the equilibrium coalitions and the division of coalitional payoxs. We believe that our work also sheds light on these models of noncooperative coalitional bargaining by analyzing (in the restricted context of simple games) the relation between the protocol and the coalitions formed in equilibrium.

Finally, our model bears a more distant relationship to the study of agenda control in binary voting surveyed in Rosenthal (1990) and Miller (1995). The description of the role of the agenda setter in those models is quite dixerent from ours. In binary voting, the agenda setter has the ability to choose the proposal to be pitted against the status quo (as in the seminal work of Romer and Rosenthal (1978)) or the complete sequence of binary votes (as in the tournament context of Miller (1980) and Banks (1985)). Once a pair of alternatives has been chosen, agents vote between the two alternatives. As in our work, the main objective of the literature is to characterize the set of outcomes which can be obtained for any agenda, and study the strategic role of the agenda setter. However, dixerences in the models predude a direct comparison between the ...ndings of the literature on binary voting and our results.

The rest of the paper is organized as follows. In Section 2, we discuss fully the power of the head of state in the formation of a cabinet with ..ve parties or less. In Section 3, we introduce our general framework and derive, for any .xed ordering of proposers, the subgame perfect equilibrium of the noncooperative mode of coalition formation. In Section 4, we analyze the power of the agenda setter and prove our main theorem, characterizing the decisive structures under which the agenda setter can fully manipulate the 
outcome of the procedure of coalition formation. The last Section contains our conclusions, and a discussion of the limitations of our analysis.

\section{Cabinet Formation with 3, 4 and 5 Parties}

To illustrate the problem of the agenda setter in coalition formation, we analyze in this section the role of the head of state in the formation of a cabinet with three, four and ..ve parties. At the beginning of the game, the head of state chooses the sequence in which the dixerent party leaders attempt to form a government. ${ }^{1}$ We suppose that each party leader has an opportunity to form thegovernment, and that no party can becalled twicein the sequence ${ }^{2}$ When a party leader is recognized to form a government, she makes an oxer to the other parties to join in a government. If all prospective members accept the oxer, the government is formed and the game ends. If at least one of the coalition members rejects the oxer, the game continues, and the next party in the sequence is recognized to form the government. If all oxers have been rejected at the end of the sequence, all parties obtain their status quo outcome.

We suppose, as in Baron and Ferejohn (1989), that parties' preferences only depend on their share in the government bene.ts, and not on the policy

\footnotetext{
${ }^{1}$ We assume that the head of state commits to the entire sequence of moves at the beginning of the game Alternatively, one could consider that the head of state only chooses the identity of the next proposer after an attempt to form the governement has failed. To model this situation, the head of state has to be treated as a player in the sequential game, who can condition his choice on the past history. This would lead to a dixerent mode, which is more complex to analyze, and does not allow for a simple description of the relation between the order of moves and the outcome of bargaining.

${ }^{2} \mathrm{As}$ long as the sequence of moves if ..nite, the assumption that no party moves twice in the game is made without loss of generality. (Se the discussion in the last Section of the paper.)
} 
chosen by the government. ${ }^{3}$ Hence, a proposal to form the government only entails a division of the government bene.ts among the parties. To simplify the analysis, we assume that the government bene.ts are equal to one for all the possible coalitional governments, and that the status quo outcome is identically equal to zero for all the parties.

The only source of heterogeneity among parties in our model thus stems from the distribution of seats in parliament. Letting $w_{i}$ denote the number of seats of party $i$ in parliament, a coalitional government $C$ can be formed if and only if ${ }_{i 2}{ }_{12} W_{i}, \frac{{ }^{P}}{2}$ : Disregarding the uninteresting case where one party obtains a majority of seats in parliament, or when some parties are "dummy players", who do not play a signi..cant role in parliament, we can easily characterize the decisive structures with three, four and ..ve players. Von Neumann and Morgenstern (1953, pp. 457-462) were the .r.st to note that, while there are of course a large number of possible distributions of seats in parliament, there are only a few prototypical decisive structures which represent the distribution of power among the parties. To characterize these decisive structures, we recall the de.nition of a weighted quota game. A weighted quota game is de .ned by a list $\left[q ; w_{1} ; w_{2} ;: ; w_{n}\right]$ where $q$ denotes the quota, $w_{i}$ the weight of player $i$ and a coalition $C$ is winning if and only if $\mathrm{P}$

i2c $W_{i}$, q. Von Neumann and Morgenstern (1953, pp. 457-462) show that there are only six weighted quota games representing the decisive structures for less than ..ve players.

For $n=3$, the structure $[2 ; 1 ; 1 ; 1]$

For $n=4$, the structure $[3 ; 2 ; 1 ; 1 ; 1]$

For $n=5$, the structures $[3 ; 1 ; 1 ; 1 ; 1 ; 1] ;[4 ; 2 ; 2 ; 1 ; 1 ; 1] ;[4 ; 3 ; 1 ; 1 ; 1 ; 1]$;

$[5 ; 3 ; 2 ; 2 ; 1 ; 1]$ :

\footnotetext{
${ }^{3}$ We discuss in the conclusion the extension of our model to a situation where parties' preferences depend on policy outcomes.
} 
With thre players, the only decisive structure is the simple majority case, where two players out of three are needed to form the government. The structures $[3 ; 2 ; 1 ; 1 ; 1]$ and $[4 ; 3 ; 1 ; 1 ; 1 ; 1]$ are examples of apex games where there exists a single large player, who can form a winning coalition with any of the small players. The structure $[3 ; 1 ; 1 ; 1 ; 1 ; 1]$ is a symmetric majority game, where all players are identical and a majority is formed by more than a half of the players. The other two structures, $[4 ; 2 ; 2 ; 1 ; 1 ; 1]$ and $[5 ; 3 ; 2 ; 2 ; 1 ; 1]$ distinguish between large, middle and small players, and represent more complex distributions of power among the parties.

As a .r.st step in the analysis, we need to determine, for every possible ordering of theparties, the coalitions formed in equilibrium. Wewill illustrate the basic principles with the case of three parties. Clearly, the last party in the sequence can extract the entire surplus by paying the other parties their status quo outcome of 0 . The second party in the sequence thus should never propose to form a coalition with the last party, but should instead propose to form a coalition with the.r.rst party, paying the .r.st party its continuation payox of 0 , and keeping a surplus of 1 . Hence, at the beginning of the game, the .r.st party never proposes to form a coalition with the second party, but forms instead a coalition with the last party. ${ }^{4}$

The same reasoning can be applied to compute the coalitions formed in equilibrium with four parties. Reorder the parties, so that party 1 is thelarge party and 2, 3 and 4 are the small parties. Barring symmetries between the small parties, there are four distinct orderings to consider. Consider ..r.st the ordering $1 ; 2 ; 3 ; 4$. At the last stage of the game, party 4 obtains the entire surplus. Hence party 3 should form a coalition excluding the fourth party,

\footnotetext{
'This result has also been obtained by Austen Smith and Banks (1988) when parties' preferences are de.ned over government bene.ts and policy outcomes. In their mode, the coalition is formed by the largest party (the .r.st player in the game) and the smallest party (the last player in the game).
} 
namely coalition $f 1 ; 3 g$ or coalition $f 1 ; 2 ; 3 g$. Party 2 then should choose to form a coalition with party 1 or parties 1 and 4 . At the beginning of the game, party 1 thus either forms the coalition $f 1 ; 3 g$ or $f 1 ; 4 g$ or $f 1 ; 3 ; 4$. Next consider the ordering $2 ; 1 ; 3 ; 4$. Party 4 again obtains the entire surplus, so that party 3 should form either the coalition $f 1 ; 3 g$ or $f 1 ; 2 ; 3 g$. Party 1 thus selects to form coalition $f 1 ; 2 g$, coalition $f 1 ; 4 g$ or coalition $f 1 ; 2 ; 4 g$. Party 2 then forms a coalition excluding party 1 , namely the coalition of small parties $\mathrm{f} 2 ; 3 ; 4 \mathrm{~g}$. If now the ordering is $2 ; 3 ; 1 ; 4$, at the last stage of the game, party 4 still captures the entire surplus. Hence party 1 forms any of the three coalitions $f 1 ; 2 g, f 1 ; 3 g$ or $f 1 ; 2 ; 3 g$. Party 3 then chooses to form the coalition $f 2 ; 3 ; 4$, and party 2 forms a coalition excluding party 3 , namely any of the coalitions $f 1 ; 2 g$ or $f 1 ; 2 ; 4 g$. Finally, if the ordering is $2 ; 3 ; 4 ; 1$, party 1 captures the entire surplus at the last stage of the game. Hence party 4 forms $f 2 ; 3 ; 4 g$. Party 3 then forms either $f 1 ; 3 g$ or $f 1 ; 2 ; 3 g$ and party 2 forms either $f 1 ; 2 g$ or $f 1 ; 2 ; 4 g$.

The analysis of the coalitions formed at equilibrium for $n=3$ and $n=4$ shows that at each stage of the game, the party which is recognized can capture the entire surplus, by forming a winning coalition which excludes the next party in the sequence. Hence, in equilibrium, the .r.st party forms any winning coalition excluding the second party. This result shows that the only relevant part of the sequence of moves chosen by the head of state are the identities of the .r.st and second parties. Hence, when computing the equilibrium coalition, we can restrict our attention to the identities of the .r.st two parties in the sequence

We now consider the coalitions formed with 5 parties in the dixerent decisive structures. In the symmetric structure $[3 ; 1 ; 1 ; 1 ; 1 ; 1]$, all orderings are equivalent. If parties 1 and 2 are the .r.st two parties, the equilibrium minimal winning coalitions are $1 ; 3 ; 4 g, f 1 ; 3 ; 5 g$ and $f 1 ; 4 ; 5 g$ and $f 1 ; 3 ; 4 ; 5 g$. Consider now the structure with two large parties and thre small parties, 
[4;2;2;1;1;1]. Let 1 and 2 denote the large parties and 3,4 and 5 the small parties. Ignoring symmetries, there are four orderings of the ..r.st two parties to consider: either the large parties are placed on top, or one large and one small party, or one small and one large party, or two small parties. The following table summarizes the coalitions formed in the four cases.

\begin{tabular}{|ll|}
\hline order & equilibrium coalition \\
12 & $134 ; 135 ; 145 ; 1345$ \\
13 & $12 ; 145 ; 124 ; 125 ; 1245$ \\
31 & $234 ; 235 ; 2345$ \\
34 & $123 ; 135 ; 235 ; 1235$ \\
\hline
\end{tabular}

In the apex game, with party 1 denoting the large party, there are three orderings of the.r.r.st two parties to consider: either party 1 is .r.rst, or a small party is .r.st and party 1 second, or the .r.st two parties are small parties.

\begin{tabular}{|ll|}
\hline order & equilibrium coalition \\
12 & $13 ; 14 ; 15 ; 134 ; 135 ; 1345$ \\
21 & 2345 \\
23 & $12 ; 124 ; 125 ; 1245$ \\
\hline
\end{tabular}

Finally, in the structure $[5 ; 3 ; 2 ; 2 ; 1 ; 1]$, we let 1 denote the large party, 2 and 3 the middle parties and 4 and 5 the small parties. There are now 8 dixerent orders of the ..rst two parties to consider. 


\begin{tabular}{|ll|}
\hline order & equilibrium coalition \\
12 & $13 ; 145 ; 1345$ \\
14 & $12 ; 13 ; 125 ; 135$ \\
21 & $234 ; 235 ; 2345$ \\
23 & $12 ; 124 ; 125 ; 1245$ \\
24 & $12 ; 123 ; 125 ; 1235$ \\
41 & $234 ; 2345$ \\
42 & $145 ; 134 ; 1345$ \\
45 & $124 ; 134 ; 1234 ; 234$ \\
\hline
\end{tabular}

These tables can now be used to analyze the power of the agenda setter in the dixerent decisive structures. It is obvious that the head of state can al ways choose one party to beincluded in the coal ition (the . r.st in the order) and one party to be exduded in the coalition (the second in the order). Can the head of state impose the presence of other parties in the government? The answer to this question depends crucially on the decisive structure of the game For example, consider the symmetric structure with ..ve parties, $[3 ; 1 ; 1 ; 1 ; 1 ; 1]$. In that symmetric majority game, the head of state can never guarante the presence of more than one party in the government. If party 1 is recognized ..r.st, she may form a coalition with either parties 3 and 4, 4 and 5 or 3 and 5 . There is no way to force the presence of either of those three parties in the government. By contrast, consider the structure with one large party and four small parties, $[4 ; 3 ; 1 ; 1 ; 1 ; 1]$. This game has ..ve minimal winning coalitions: $f 1 ; 2 g ; f 1 ; 3 g ; f 1 ; 4 g ; f 1 ; 5 g$ and $f 2 ; 3 ; 4 ; 5 g$. We claim that the head of state can guarante the presence of any minimal winning coalition in the government. In order to guarantee a coalition with two parties, $f 1 ; 2 g$, the head of state can choose any order placing 2 ..r.st and 1 in third, fourth or ...f.th position. In order to guarantee the formation of 
the coalition of small parties, the head of state should place the large party second. In the structure with two large parties and three small parties, the head of state can guarantee the presence of one small party and one large party in the coalition, say parties 2 and 3, by placing party 3 .r.st and party 1 second, but cannot guarantee the presence of any other group of parties in the equilibrium coalition. In the structure with one large party, two middle parties and two small parties, $[5 ; 3 ; 2 ; 2 ; 1 ; 1]$, the head of state can guarantee the presence of one large and one middle party, say parties 1 and 2, by placing party 2 .r.st and party 3 second. He can also impose the presence of the minimal winning coalition consisting of the two middle parties 2 and 3 and one of the small parties, player 4 , by placing party 4 .r.st and party 1 second. However, the head of state cannot force the presence of the minimal winning coalition $f 1 ; 4 ; 5$ in the government: there exists no ordering of the parties which guarantees that $f 1 ; 4 ; 5 \mathrm{~g}$ al ways belongs to the equilibrium coalition.

The study of government formation with ..ve parties or less thus shows that the agenda setter's ability to impose the presence of a player in the equilibrium coalition is related to the underlying decisive structure of the game. In the next sections of the paper, we consider a general model of coalition formation in simple games, and investigate fully the relation between the power of the agenda setter and the underlying simple games.

\section{Coalition Formation in Simple Games}

We consider a TU cooperative game $(\mathrm{N} ; \mathrm{v})$ where $\mathrm{N}$ is the set of players and $v$ a coalitional function, assigning to each nonempty subset $\mathrm{C}$ of $\mathrm{N}, \mathrm{a}$ real number $v(C) 2<_{+}$. Since our aim is to study the power of the agenda setter in the determination of the coalition formed, rather than the division 
of payous among coalition members, we focus in this paper on simple games. Recall that a cooperative game is simple if and only if $v(C) 2 \mathrm{f} 0 ; \mathrm{g}$. A coalition $C$ is called winning if $\mathrm{v}(\mathrm{C})=1$ and losing if $\mathrm{v}(\mathrm{C})=0$.

We .r.st recall some well known properties of simple games (see Weber (1994), p.1291). A simple game is monotone if $\left.v(C)=1 ; C_{1 / 2} D\right) \quad v(D)=$ 1. For any monotone simple game, we can de. ne minimal winning coalitions as the smallest winning coalitions of the game $A$ simple game is proper if $v(C)=1) \quad v(\bar{C})=0$, where $\bar{C}$ denotes the complement of the set $C$. A simple game is strong if $\mathrm{v}(\mathrm{C})=0) \mathrm{v}(\overline{\mathrm{C}})=1$. A simple game is decisive if it is proper and strong. Player $i$ is called a veto player if she belongs to all winning coalitions. Player $i$ is more desirable than player $j$ if and only if, for any coalition $C$ excluding $i$ and $j, v(C[f j g)=1) \quad v(C[f i g)=1$ : Finally, a player $i$ is a dummy player if she does not belong to any minimal winning coalition.

The procedure of coalition formation that we consider is a simple extensive form noncooperative game inspired by recent models of coalitional bargaining (Chatterjee et al. (1993), Bloch (1996), Ray and Vohra (1999)). Players are ordered according to $a$..xed protocol $1 / 2$ Player $i$ is called the $1 / 2 k$ th player if $1 /(1 \mathrm{i})=\mathrm{k}$ : The $1 / 2$.r.st player starts the game by proposing a coalition $\mathrm{C}$ to which she belongs and a division of the coalitional payous among the members of $C$, namely a vector of payous $f y_{i} g_{i 2 c}$ such that $y_{i}, 0$ for all $i$ and ${ }_{i 2 C} y_{i}=1$ : All the members of $C$ respond to the oxer in the ordering speci. .ed by $1 / 2$ If all members accept the oxer, the coalition is formed, and players obtain payoas $x_{i}=y_{i}$ if i $2 C, x_{i}=0$ if $i z C$. If at least one of the members rejects the oxer, the $1 / 2$ second player makes a proposal. If the proposal of the second player is rejected, the third player makes a proposal. The game continues in that way until the $1 / 2$ last player makes a proposal. If her proposal is in turn rejected, all players obtain their status quo payox, 
$x_{i}=a_{i}$ with $a_{i}, 0$ and ${ }^{P} a_{i}<1::^{5}$ Since the noncooperative game depends on the underlying simple game $v$ and the protocol $1 / 2$ we denote it by $G(v ; 1)$ :

While this game of coalition formation embodies strong assumptions on the bargaining procedure, we have adopted it because it is the simplest and most transparent game wherethe protocol matters. Asthegameis . .nite, and the ordering of moves is deterministic, the payoxs of players are completely determined by the protocol. It is this feature of the game that we emphasize here, because we focus on the relationship between the protocol and the coalitions formed in equilibrium.

A subgame perfect equilibrium of the game of coalition formation is a sequence of proposals and responses such that, at any point in the game, all players act optimally. An equilibrium coalition is a coalition formed at a subgame perfect equilibrium of the game. We are now ready to characterize the equilibrium coalitions for any protocol $1 / 2$ and any simple game $v$ without veto players.

P roposition 1 Suppose that the game $v$ is monotone and does not have any veto player. Then any coalition containing the $1 / 2$. rst player and excluding the $1 / 2$ second player is an equilibrium coalition of $G(v ; 11$. Furthermore, the $1 / 2$.r.st player captures the entire coalitional surplus.

Proof. Let $k=1 ; 2:: ; n$ denote the $1 / 2 k$ th player. For any subgame perfect equilibrium, we denote by $C^{k}$ the coalition proposed by the kth player in equilibrium. We will show by induction that, for any subgame perfect

\footnotetext{
In the political interpretation, the status quo payox represents the payoo of the parties in a caretaking government, or when new elections are called. The speci..cation of a vector of status quo payment also allows us to extend the analysis to an in. .nite horizon game where the ..nite sequence of oxers is repeated. The status quo payoxs can then be interpreted as the continuation payous of the players in a stationary perfect equilibrium of the game, and the analysis of the game remains unchanged.
} 
equilibrium of the game, for any $k=1 ; 2 ;:: n ; 1$, the coalition formed by the kth player excludes player $k+1$, i.e. $(k+1) z C^{k}$ :

We .r.st de ne the continuation payo that player i obtains after the oxer of player $k$ is rejected, $u_{i}^{k}$ : If the oxer of player $n$ is rejected, all players obtain their status quo payox, so $u_{i}^{n}=a_{i}$. Consider now the second to last player, player $\mathrm{n} i \mathrm{l}$ and let $\mathrm{S}$ and $\mathrm{T}$ be two winning coalitions containing player $n i 1$ with $n z S$ and $n 2 T$. (It is al ways possible to ..nd a winning coalition containing $n_{i} 1$ and excluding $n$ since $N$ nf ng is winning and the game is monotone) The minimal amount that player $n_{i} 1$ must give to his coalition partners in coalitions $S$ and $T$ is given by ${ }^{P}{ }_{i 2 S ; i \epsilon n_{i} 1} u_{i}^{n_{i}}{ }^{1}$ and $i 2 T ; i n_{i} 1 u_{i}^{n_{i} 1}$ respectively. Now, clearly, $u_{i}^{n_{i} 1}=0$ if $i z C^{n} ; u_{i}^{n_{i} 1}=a_{i}$ if i $2 C^{n}$; i $\in n$ and $u_{n}^{n_{i}{ }^{1}}=1_{i} \quad$ i $2 C^{n} ; i \in n a_{i}$ : We thus compute

$$
\begin{aligned}
& X \quad u_{i}^{n_{i} 1}=X \quad a_{i}
\end{aligned}
$$

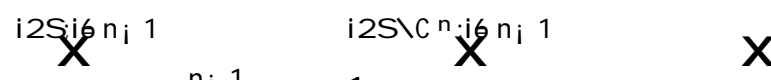

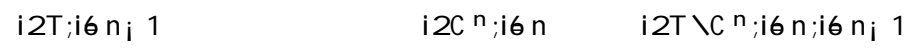

Next observe that since $T$ is a winning coalition containing player $n$, player $n$ could have chosen to form $T$. Hence

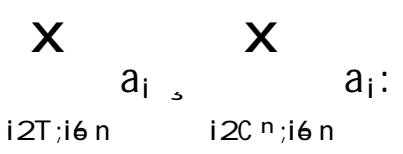

But

$$
\begin{aligned}
& x \quad x \quad x
\end{aligned}
$$

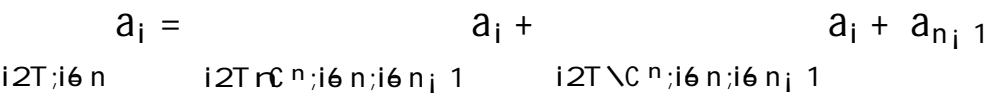

So

$$
X \underset{i 2 T ; i \epsilon n_{i} 1}{u_{i}^{n_{i} 1}, 1_{i}} \underset{i 2 T n C n ; i \epsilon n ; i \epsilon n_{i} 1}{X} a_{i} i a_{n_{i} 1}
$$

Now

$$
\underset{i 2 T n C^{n} ; i \epsilon n ; i \epsilon n_{i} 1}{X} a_{i}+a_{n i 1}+{ }_{i 2 S \backslash C^{n} ; i \epsilon n_{i} 1}^{X} a_{i 2 N} \cdot a_{i}<1 ;
$$


so we ..nally obtain

$$
\begin{aligned}
& \begin{array}{l}
X \quad u_{i}^{n_{i} 1}> \\
u_{i}^{n i} 1 \text {; }
\end{array} \\
& \text { i2T;i } i n_{i} 1 \quad i 2 S ; i \Leftrightarrow n_{i} 1
\end{aligned}
$$

showing that, in any subgame perfect equilibrium of the game, player $n$ i 1 forms a coalition which excludes player $n$.

Consider now the general step, and suppose that for all $t>k$, in all subgame perfect equilibria of the game, $t+1 z \mathrm{C}^{\mathrm{t}}$ : Consider again two winning coalitions containing player $\mathrm{k}, \mathrm{S}$ and $\mathrm{T}$ with $\mathrm{k}+1 \mathrm{zS}$ and $\mathrm{k}+12 \mathrm{~T}$ : The minimal amount that player $\mathrm{k}$ must give to his coalition partners in coalitions $S$ and $T$ is given by ${ }_{i 2 S ; i \epsilon k} u_{i}^{k}$ and $P$ i2T;iєk $u_{i}^{k}$ respectively. Notice that, for any $t, k$, the continuation payous of the players are given by the recursive expressions: $u_{i}^{t}=0$ if i $z C^{t+1}, u_{i}^{t}=u_{i}^{t+1}$ if i $2 C^{t+1}$ and i $\theta t+1$ and $u_{t+1}^{t}=1_{i} \quad P \quad i 2 C^{t+1 ; i \epsilon t+1} u_{i}^{t+1}$ : Hence we obtain

$$
\begin{aligned}
& X \quad u_{i}^{k}=X \quad u_{i}^{k+1} \\
& \stackrel{i 2 S ; i \epsilon k}{X^{k}}=1 i{ }^{i 2 S \backslash C^{k+1} \cdot j \notin k} u_{i}^{k+1}+\quad X \quad u_{i}^{k+1} \\
& \text { i2T;i } \quad \text { i2k } C^{k+1 ; i \epsilon k+1} \quad \text { i2T } \backslash C^{k+1} ; i \epsilon k ; i \epsilon k+1
\end{aligned}
$$

Since player $k+1$ could have chosen to form the coalition $T$,

$$
\underbrace{}_{i 2 T ; i \epsilon k+1} u_{i}^{k+1},{ }_{i 2 C^{k+1} ; i \epsilon k+1}^{X} u_{i}^{k+1}:
$$

Now notice that

$$
X \underset{i 2 T ; i \epsilon k+1}{X} u_{i}^{k+1}=\underset{i 2 T n C^{k+1} ; i \epsilon k ; i \epsilon k+1}{X} u_{i}^{k+1}+{ }_{i 2 T \backslash C^{k+1} ; i \epsilon k ; i \epsilon k+1}^{X} u_{i}^{k+1}+u_{k}^{k+1}
$$

Hence,

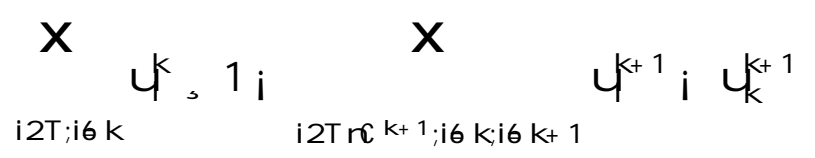


and

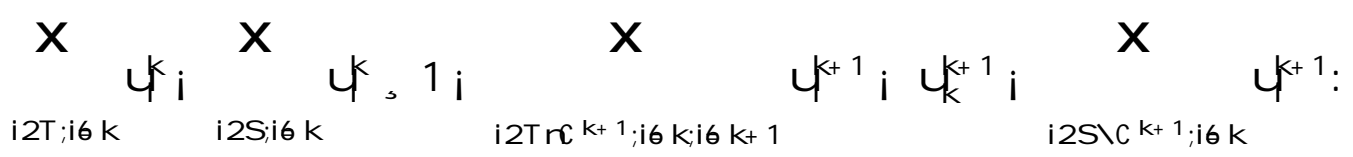

To .. nish the proof, two cases must be distinguished. If $k+2 z T$, then $P^{i 2 T n C^{k+1} ; i \epsilon k ; i \epsilon k+1} u_{i}^{k+1} . P{ }_{i 2 T n C^{k+1} ; i \epsilon k ; i \epsilon k+1} a_{i} ; u_{k}^{k+1} \cdot a_{k}$ and ${ }^{P}{ }_{i 2 S \backslash C^{k+1} ; i \epsilon k} u_{i}^{k+1}$. i2sı $c^{k+1} ; i \in k a_{i}$. Hence

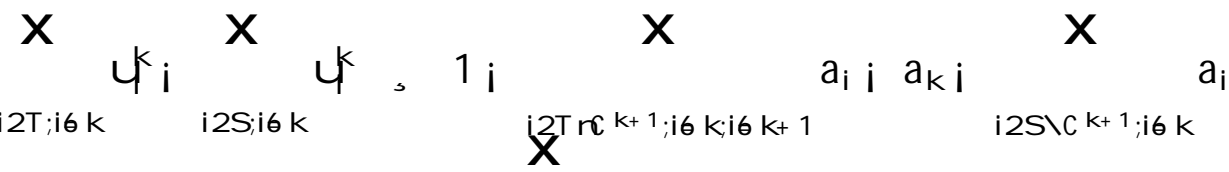

$$
\begin{aligned}
& \text {, } 1 i \quad a_{i}>0 \text { : } \\
& \text { i2 N }
\end{aligned}
$$

If $k+22 T$, player $k+1$ must strictly prefer the coalition $C^{k+1}$ to the coalition $T$. Hence,

$$
{ }_{i 2 T ; i \epsilon k+1} u_{i}^{k+1}>{ }_{i 2 C^{k+1} ; i \epsilon k+1}^{X} u_{i}^{k+1}
$$

and

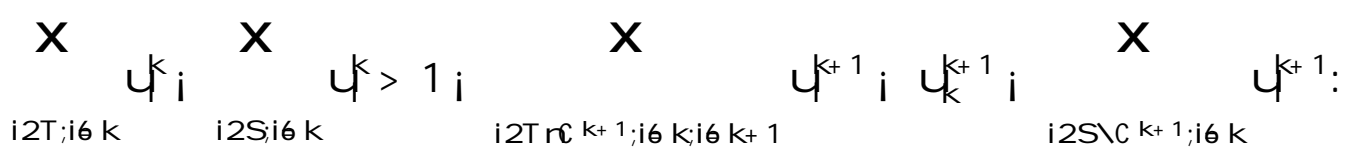

Now,

$$
\begin{aligned}
& 1_{i} \quad \begin{array}{lllll}
\mathrm{i} & \mathrm{X} & \mathrm{u}_{\mathrm{i}}^{\mathrm{k}+1} \mathrm{i} & \mathrm{u}_{\mathrm{k}}^{\mathrm{k}+1} \mathrm{i} &
\end{array} \\
& =1 i^{i 2 T n C^{k+1} ; i \notin k i i \epsilon k+1} u_{i}^{k+1} i\left(1 i^{i 2 S \backslash C^{k+1 ; i i k k}} u_{i}^{k+2}\right) i u_{k}^{k+1} i \\
& \dot{X}^{\top 2^{T n C^{k+1}} ; i \epsilon k ; i \epsilon k+1 ; k+2} \quad x^{i 2 C^{k+2} ; i \epsilon k+2} \quad x^{i 2 S \backslash C^{k+1} ; i \epsilon k}
\end{aligned}
$$

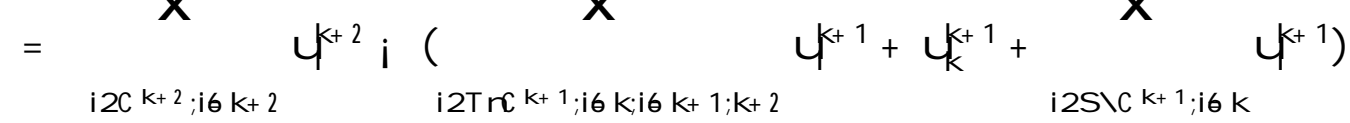


Now, since $k+2 z C^{k+1}$ and $u_{i}^{k+2}, u_{i}^{k+1}$ for all $i \xi k+2$,

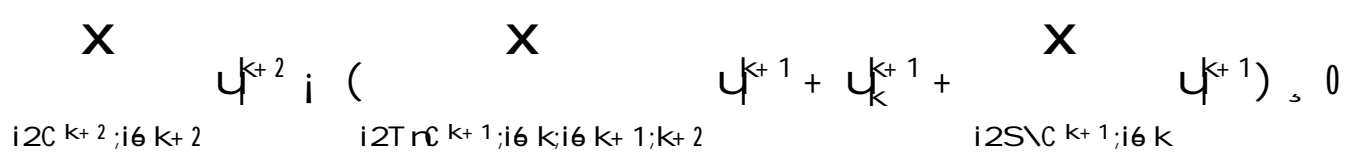

so that

$$
{ }_{i 2 T ; i \epsilon k} u_{i}^{k} i \underset{i 2 S ; i \epsilon k}{X} u_{i}^{k}>0
$$

establishing the induction result. To complete the proof, note that player 1 always forms a coalition excluding player 2 and that for all $k, 3, u_{k}^{1}=0$. So player 1 is indixerent among all the winning coalitions which exdude player 2 and is able to capture the entire surplus.

Proposition 1 completely characterizes the subgame perfect equilibria of the procedure of coalition formation, when the simple game does not have any veto player. All subgame prefect equilibria induce the same distribution of payous, with the .r.st player capturing the entire surplus. ${ }^{6}$ The logic underlying the result is that in equilibrium, at any stage of the game, a player oxers a coalition which excludes her immediate successor. Running the argument backwards, it appears that at the beginning of the game, player 1 should al ways exclude player 2 . Furthermore, since each player $k$, 3 has been excluded from the coalition formed by its immediate predecessor, her continuation payox in the game is zero, and hence the .r.st player captures the entire coalitional surplus.

\footnotetext{
${ }^{6}$ Notice that, in any subgame perfect equilibrium of the game, all the players except the ..rst one play weakly dominated strategies. They accept an oxer of 0 immediately, precluding the possibility of obtaining a positive payox at later stages in the game. While these strategies may seem unnatural, they are the only strategies consistent with subgame perfect equilibrium play.
} 
The sequential structure of the game thus yields a very unequal distribution of the surplus among the players. In order to mitigate this problem, we could have assumed that every player has an outside option " $i$ and must obtain at least her outside option to participate in the government. For a vector of " $i$ 's small enough, the thrust of the analyis remains unchanged, and in equilibrium, all players must exclude their immediate successor in the coalition they form. With a vector of outside options, the equilibrium coalition formed by the .r.st player becomes determinate: it is the winning coalition excluding player 2 and containing the players with the smallest outside option. Notice in particular, that in this slightly perturbed variant of the game, the equilibrium coalition is al ways a minimal winning coalition.

The main diф culty in the proof of Proposition 1 stems from the existence of an arbitrary status quo payou for the players. However, it is striking to note that the equilibrium distribution of the surplus is independent of the status quo and only depends on the ordering of the players. This result is obtained because each player has an opportunity to make an oxer, and hence each player is excluded from a coalition at least once in the game, so that her continuation payox is equal to zero and independent of the status quo. If the protocol did not let all players make an oxer, this result would not hold. Some players may never be excluded from the coalition formed, and thus obtain at least their status quo payox.

The equilibrium characterization of Proposition 1 depends crucially on the assumption that the game $v$ does not have any veto player, so that any player can form a winning coalition excluding her immediate successor. If the simple game $v$ admits veto players, the subgame perfect equilibria of the game are not easily characterized and depend on the status quo payoxs. It appears that since veto players belong to winning coalitions, they must earn at least their status quo payoo in equilibrium. The last veto player in the game can secure for herself a large share of the surplus (one minus 
the minimum sum of status quo payoxs of the other players in a winning coalition), and other veto players can similarly obtain a share of the surplus larger than their status quo payox. Depending on the order of veto and nonveto players and the structure of thegame, some nonveto players can also obtain a positive share of the surplus. Overall, the equilibrium coalitions and equilibrium payoas depend in a complex way on the ordering of players and the status quo outcomes.

The only case where the equilibrium of a game with veto players can easily be characterized is when all status quo payous are equal to zero. In that case, the last veto player captures the entire surplus. Le $k$ denote that last veto player. It is easy to see that in equilibrium, the continuation payox obtained by the players after the oxer of player $\mathrm{k}$ has been rejected are 0 for all i $\in k+1$ and 1 for player $k+1$. Since however, by assumption, player $k+1$ is not a veto player, player $k$ can form a winning coalition excluding player $k+1$, and propose 0 to all coalition members. Hence, when it is player k's turn to make an oxer, she can secure for herself a payment of 1 . Next, since player $k$ is a veto player, she must belong to any winning coalition, and hence any player $j$ making oxers before player $k$ must oxer her continuation payox of 1.

The fact that the last veto player obtains the entire surplus is hardly surprising given the sequential structure of the procedure we analyze. When there are veto players, our game can be construed as a simple extension of an ultimatum game (when all players are veto players, our game is exactly an ultimatum game), and the last player can thus obtain the entire surplus.

\section{A genda Control and Coalitions}

In this section, we analyze the power of an agenda setter who chooses the order $1 / 2$ in which players make oxers in the noncooperative game of coalition 
formation. We focus here on games without veto players. By Proposition 1, the only relevant part of the protocol $1 / 2$ is the choice of the .r.st two players.S Proposition 1 also clearly shows that an agenda setter can al ways impose the presence of one of the players (player 1) and exclude another player (player 2) from the equilibrium coalition. We are thus interested in situations where the agenda setter wants to impose the presence of more than one player in the equilibrium coalition.

De..nition 2 The agenda selter can imposethepresence of a set $S$ of players in the game $v$ if there exists an ordering $1 / 2$ such that the set $S$ belongs to all equilibrium coalitions of the game $G(v ; 1 / 2$.

Using the characterization result of Proposition 1, we can prove the following Proposition.

P roposition 3 Let $v$ be a monotone game without veto players. The agenda setter can impose the presence of a set $\mathrm{S}$ of players in the game $v$ if and only if there exists a player i $2 \mathrm{~S}$ and a player $\mathrm{j} Z \mathrm{~S}$ such that for all $\mathrm{k} 2 \mathrm{~S} ; \mathrm{k} \in \mathrm{i}$, $\mathrm{v}(\mathrm{Nnf} ; \mathrm{kg})=0$. To impose the presence of the set $S$, the agenda setter chooses ${ }^{1} /(k i)=1 ;{ }^{1} /(j)=2$ :

P roof. Suppose that the condition is satis. ed, and consider an ordering where i is placed .r.st and $\mathrm{j}$ is placed second. By Proposition 1, any winning coalition containing $i$ and excluding $j$ is an equilibrium coalition. Now, if there exists $\mathrm{k} 2 \mathrm{~S} ; \mathrm{k} \in \mathrm{i}$ such that $\mathrm{k}$ does not belong to an equilibrium coalition, there must exist a winning coalition excluding $j$ and $k$, contradicting the assumption.

Suppose now that the condition is violated, and consider any ordering of the players. Let $\mathrm{i}$ and $\mathrm{j}$ be the .r.st and second players in that ordering. If 
j $2 \mathrm{~S}$, by Proposition 1, j does not belong to the equilibrium coalition, and hence the agenda setter cannot impose the presence of $S$ : If now $j z S$, since the condition is violated, there exists $\mathrm{k} 2 \mathrm{~S} ; \mathrm{k} G \mathrm{i}$ such that $\mathrm{v}(\mathrm{N} \mathrm{nf} ; \mathrm{kg})=1$. Hence, by Proposition $1, \mathrm{~N} \mathrm{nf}$; $\mathrm{kg}$ is an equilibrium coalition, and the agenda setter cannot impose the presence of $\mathrm{S}$.

Proposition 3 provides a simple criterion to check whether a coalition can be imposed by the agenda setter. In the de. nition of the criterion, two players play a particular role: a member of the coalition i who is placed .r.st by the agenda setter, and a player outside the coalition, player $j$, who is placed second in the order. It is easy to see that, if players can be ordered by the desirability relation, in order to meet the condition, player i should be selected as the least desirable member of the coalition, and player $j$ as the most desirable player outside the coalition. Formally,

Proposition 4 Let $v$ be a monotone game without veto players. If the agenda setter can impose the presence of a set $\mathrm{S}$ of players by choosing $1 /(k \mathrm{i})=1$ and ${ }^{1} /(\mathrm{j} j)=2$, and if $\mathrm{i}^{0} 2 \mathrm{~S}$ is less desirable than $\mathrm{i}$ and $\mathrm{j}^{0} \mathrm{ZS}$ is more desirable than $j$, then the agenda setter can impose the presence of the set $\mathrm{S}$ by choosing $1 / 2 \mathrm{i} 9=1$ and $1 / 6 \mathrm{j} 9=2$ :

P roof. Since the agenda setter can impose the presence of $S$ by placing $i$ .r.st and $\mathrm{j}$ second, $8 \mathrm{k} 2 \mathrm{~S} ; \mathrm{k} \in \mathrm{i} ; \mathrm{v}(\mathrm{N} \mathrm{nfj} ; \mathrm{kg})=0$. To prove the proposition, we simply need to show that $8 \mathrm{k} 2 \mathrm{~S} ; \mathrm{k} \in \mathrm{i}^{0}, \mathrm{v}\left(\mathrm{N} \mathrm{nf} \mathrm{j}^{0}, \mathrm{~kg}\right)=0$. For all $\mathrm{k} 2$ $\mathrm{S} ; \mathrm{k} \in \mathrm{i} ; \mathrm{v}\left(\mathrm{fj} \mathrm{g}^{\mathrm{g}}\left[\mathrm{Nnf} ; \mathrm{j}^{\mathrm{a}}, \mathrm{kg}\right)=0\right.$. Since ${ }^{0}$ is more desirable than $\mathrm{j}, \mathrm{v}(\mathrm{fj} \mathrm{g}[$ $\left.\mathrm{Nnfj} ; \mathrm{j}^{0}, \mathrm{~kg}\right)=\mathrm{v}\left(\mathrm{Nnfj}^{0}, \mathrm{~kg}\right)=0$. In particular, $\mathrm{v}\left(\mathrm{N} \mathrm{nfj}^{0} ; \mathrm{ig}\right)=\mathrm{v}(\mathrm{fig}[$ $\left.\mathrm{Nnf} i ; i^{0}, j g\right)=0$. Since $i$ is more desirable than $i^{0}, v\left(f i g\left[N\right.\right.$ f $\left.i ; i^{0}, j g\right)=$ $\mathrm{v}(\mathrm{Nnf} ;$; $\mathrm{g})=0$, completing the proof of the Proposition.

Whilethe de. nition of thecriterion of Proposition 3 and of the desirability relation may be di $₫$ cult to interpret in general simple games, they are more 
transparent in weighted quota games. Recall that in a weighted quota game, player $i$ is more desirable than player $j$ if and only if $w_{i}, w_{j}$. We can now combine Proposition 3 and Proposition 4 to characterize the sets of players who can imposed by an agenda setter in weighted quota games.

Corollary 5 Let $v$ bea weighted quota game. For any coalition $C$, let $a_{C}$ and $b_{c}$ be the smallest and largest players in $C$. The agenda setter can impose the presence of a set $S$ of players if and only if $8 i 2 S$, i $G a_{S} ; w_{i}+w_{b_{5}}>1_{i} q$.

To illustrate Corollary 5, consider the present (as of December 1999) composition of the Dutch parliament.

\begin{tabular}{|ll|}
\hline party & seats \\
& \\
Social democrats (PvDA) & 45 \\
Liberals (VVD) & 38 \\
Christian Democrats (CDA) & 29 \\
Democracy 66 (D66) & 14 \\
Green Left & 11 \\
Socialist Party & 5 \\
Others & 10 \\
\hline
\end{tabular}

Since the Parliament contains 150 seats and a simple majority is needed to form the government, the quota is given by $q=76$. From Corollary 5 , it appears that the head of state can impose the presence of the Social Democrats with any other party but the Liberals, and the presence of the Liberals with any other party but the Social Democrats. These are the only groups of parties that the head of state can impose in the equilibrium coalition.

In the preceding analysis, we have characterized the set of players that the agenda setter can impose in an equilibrium coalition. Irrespective of 
the underlying game, the agenda setter can always impose the presence of one agent (the .r.st) in the winning coalition. Proposition 3 shows that, under some restrictions on the underlying simple game, the agenda setter can impose the presence of larger sets of players in the equilibrium coalition. Can the agenda setter impose the presence of all members of a winning coalition? Clearly, if a nonminimal winning coalition is an equilibrium coalition of the game, there exists a minimal winning coalition which is also obtained in equilibrium. Hence, the agenda setter can never impose the presence of all players in a winning coalition which is not minimal. We now characterize the simple games for which the agenda setter can impose the presence of any minimal winning coalition in the equilibrium coalition.

To this end, we restrict our attention to decisive games (games which are both proper and strong) and we exclude dummy players. A decisive game $v$ is called an apex game if there exists a player $i$ such that $v(f i ; j)=1$ for all $\mathrm{j}$ in $\mathrm{N}$. We now state our main Theorem.

Theorem 6 Let $v$ be a decisive simple game without veto players nor dummy players. The agenda setter can impose the presence of any minimal winning coalition in the equilibrium coalition if and only if $v$ is an apex game.

Proof. We .r.st show that, if $\mathrm{v}$ is an apex game, the agenda setter can impose the presence of any minimal winning coalition. Let $\mathrm{i}$ be the large player. Consider ..rst a two player coalition $S=f i ; j$. For any $k z S$, $\mathrm{v}(\mathrm{Nnfi} ; \mathrm{kg})=0$. Now consider the coalition of all the small players, $\mathrm{S}=$ $\mathrm{N}$ nfig. For any $\mathrm{k} 2 \mathrm{~S}, \mathrm{v}(\mathrm{N} \mathrm{nfi} ; \mathrm{kg})=0$. Hence the condition of Proposition 3 is satis. ed for any minimal winning coalition $\mathrm{S}$.

To prove the reverse implication, consider a game $v$ for which all minimal winning coalitions can be imposed. We will show that the game $v$ is an apex game. If $\mathrm{jNj}=3$, the result is immediate. So suppose that $\mathrm{jNj}$, 4. Since 
the game is proper, there must exist a minimal winning coalition $\mathrm{S}$ with jSj , 3. As the head of state can impose the presence of all the players of $S$ in the equilibrium coalition, there exist two players $a$ and $b$ in $S$ and a player $z Z S$ for whom $v(f a ; z g)=v(f b ; z)=1$ :

Now de.ne $Z=f i 2 \mathrm{~N} ; \mathrm{i} \in \mathrm{z} ; \mathrm{v}(\mathrm{fi} ; \mathrm{zg})=1 \mathrm{~g}$ : The set $\mathrm{Z}$ is the set of all players who are winning with $z$, and by our previous argument, jZj , 2 . Suppose that there exists a player i $2 N ; i \in z$ such that $i z Z$. Since $i$ is not a dummy player, there exists a minimal winning coalition $S_{i}$ to which she belongs. Now observe that, since the game is proper, for all a 2 $\mathrm{Z} ; \mathrm{v}(\mathrm{N} \mathrm{nf} \mathrm{a} ; \mathrm{Zg})=0$. This implies that the winning coalition $\mathrm{S}_{\mathrm{i}}$ must either contain $z$ or all the players in $Z$, i.e. either $z 2 S_{i}$ or $Z \frac{1}{2} S_{i}$. We now show that $\mathrm{v}(Z)=0$. Suppose to the contrary that $\mathrm{v}(Z)=1$. By minimality of the winning coalition $S_{i}$; we cannot have $Z 1 / 2 S_{i}$. Hence $z 2 S_{i}$. Furthermore, as $v(Z)=1$ and the game is proper, $S_{i} \backslash Z G_{;}$, implying that there exists a $2 \mathrm{Z}$ such that $f a ; z g \frac{1}{2} S_{i}$, contradicting the minimality of the winning coalition $S_{i}$.

Since $v(Z)=0$ and the game is strong, $v(N n Z)=1$. Hence there exists a minimal winning coalition $S \frac{1}{2} 2 \mathrm{~N} \mathrm{nZ}$. Clearly, since Z Ã S, z 2 S: We now claim that $\mathrm{jSj}=2$. Suppose by contradiction that $\mathrm{jSj}$, 3. Since the head of state can impose the presence of all players in $\mathrm{S}$ in the minimal winning coalition, there exists $d 2 \mathrm{~S} ; \mathrm{d} G \mathrm{z}$ and $\mathrm{y} z \mathrm{~S}$ such that $\mathrm{v}(\mathrm{fd} ; \mathrm{y}) \mathrm{g}=1$ : Now recall that $v(f a ; z g)=v(f b z g)=1$ : Since the game is proper, $d \in z ; d G$ $a ; d \in$ band $y \in z$, we must have $y=a$ and $y=b$, contradicting the fact that $a$ and $b$ are distinct elements of $Z$. We conclude that there exists a player $d z Z$ for whom $v(f d ; z g)=1$, contradicting the de. nition of $Z$.

Hence, $Z=N$ nf zg, establishing that the game is an apex game.

Theorem 6 dari..es the relationship between the agenda setter's control and the underlying structure of power. In a sense, it shows that the agenda 
setter's manipulation possibilities are rather restricted: he can only fully choose the minimal winning coalition when the underlying simple game has the speci..c structure of an apex game, with one large player and many small players. In all other situations, the agenda setter's power is very restricted. He may be able to impose some but not all minimal winning coalitions, or even not beableto imposethepresence of any more than one player, as would be the case in a symmetric majority game with more than ..ve players, where no two-player coalition is winning.

\section{Conclusion}

In the theoretical models of government formation in political science, it is usually assumed that the head of state has no strategic power. In this paper, we develop a formal model of agenda control to analyze the power of an agenda setter in the formation of coalitions in simple games. We suppose that the agenda setter can choose the order in which players make proposals to form coalitions in a simple, sequential noncooperative procedure of coalitional bargaining. We show that the agenda setter can always impose the presence of one agent in the coalition by placing him .r.st, and exclude another agent by placing him second. The only structure in which the agenda setter can impose the formation of any minimal winning coalition are apex games, where a large player forms a winning coalition with any of the other players. Furthermore, the agenda setter should always place the least desirable member of a coalition .r.st, and the most desirable player outside the coalition second.

While our results provide a .r.st step in the understanding of the relation between the order of proposers and the coalitions formed in a sequential bargaining game, we point out some important limitations of our analysis, which 
need to be addressed in future research. First, we focus in this paper on a purely redistributive model of coalition formation. In order to explain the formation of governments in parliamentary democracies, we need to extend the model to accommodate for ideological preferences of the political parties. Austen Smith and Banks (1988) and Baron (1991 and 1993) have analyzed se quential models of government formation with three parties with preferences over a policy space In Austen Smith and Banks (1988), parties' utilities al so depend on their share in the government bene.ts, and government bene.ts are assumed to be large enough to compensate a coalition member for any loss in utility due to an adverse policy choice. Hence, in equilibrium, the .r.st party chooses its optimal policy outcome, and uses a transfer in government bene. ts to induce the other coalition member to participate in the government. In essence, the assumption that government bene.ts are high enough implies that all transfers will be made through a redistribution of government bene.ts, and the equilibrium is similar to the equilibrium in the purely redistributive game. In Baron (1991 and 1993), parties have purely ideological preferences, and bargain over policy outcomes. In that case, parties' strategies at the government formation stage are much more complex to characterize, and the equilibria may involve dixerent coalitions than the ones obtained in the redistributive case The characterization of equilibrium coalitions with more than thre parties in Baron's models appears to be a formidable task.

Second, we have focussed our attention to simple games, dividing the set of coalitions into only two types, and only allowing for coalitional worths of 0 or 1. As shown in Chatterjee et al. (1993), the study of the relation between the protocol and the equilibrium coalition in general cooperative games with transferable utility is extremely complex. Chatterje et al. (1993) show that, in order for the grand coalition to be formed for any ordering of the players, the per member worth of the grand coalition must exceed the per 
member worth of any other coalition. In all other cases, the coalition formed in equilibrium may not be eф cient (i.e. may be dixerent from the grand coalition for strictly superadditive games) and may depend on the protocol. The exact link between the protocol and the coalition formed in equilibrium is however diф cult to characterize.

Third, we have considered a speci..c ..nite procedure of sequential bargaining, where each player is given the opportunity to form a coalition once. As is apparent from the characterization of the subgame perfect equilibrium of the game of coalition formation, allowing players to move more than once in the sequence would not axect our results. However, excluding some players from the order of proposers would clearly change our analysis, since the status quo payous would then play a role in the determination of the equilibrium outcome. This implies that the agenda setter could bene.t from excluding some players from the order of proposers, and that his power may be enlarged by allowing him to choose incomplete orderings of the players.

\section{References}

Austen-Smith, D. and J . Banks (1988) "Elections, Coalitions and Legislative Outcomes," American Political Science Review 82, 405-422.

Banks, J . (1985) "Sophisticated Voting Outcomes and Agenda Control," Social Choice and Welfare 2, 295-306.

Baron, D. (1991) "A Spatial Bargaining Theory of Coalition Formation in Parliamentary Systems," American Political Science Review 85, 137-164.

Baron, D. (1993) "Government Formation and Endogenous Parties," American Political Science Review 87, 34-47.

Baron, D. and J. Ferejohn (1989) "Bargaining in Legislatures," American Political Science Review 83, 1181-1206. 
Bloch, F. "Sequential Formation of Coalitions with Fixed Payox Division," Games and Economic Behavior 14, 90-123.

Chatterjee, K., B. Dutta, D. Ray and K. Sengupta (1993) "A Noncooperative Theory of Coalitional Bargaining," Review of Economic Studies 60, 463-478. Diermeier, D. and A. Merlo (1999) "An Empirical Investigation of Coalitional Bargaining Procedures," mimeo., MEDS, Northwestern University and New York University.

J ackson, M. and B. Moselle (1998) "Coalition and Party Formation in a Legislative Voting Games," mimeo., California Institute of Technology.

Laver, M. and K. Shepsle (1996) Making and Breaking Governments, Cambridge Cambridge University Press.

Merlo, A. (1997) "Bargaining over Governments in a Stochastic Environment," J ournal of Political Economy 105, 101-131.

Miller, N. (1980) "A New Solution for Tournaments and Majority Voting," American J ournal of Political Science 24, 68-96.

Miller, N. (1995) Committees, Agendas and Voting, Chur, Switzerland: Harwood Academic Publishers.

Ray, D. and R. Vohra (1999) "A Theory of Endogenous Coalition Structures," Games and Economic Behavior 26, 286-336.

Romer, T. and H. Rosenthal (1978) "Political Resource Allocation, Controlled Agenda, and the Status Quo," Public Choice 33/ 4, 27-45.

Rosenthal, H. (1990) "The Setter Mode," in Advances in the Spatial The ory of Voting, ed. by J. Enelow and M. Hinich. Cambridge: Cambridge University Press.

Strom, K. , I. Budgeand M. Laver (1994) "Constraints on Cabinet Formation in Parliamentary Democracies," American J ournal of Political Science 38, 303-335. 
Von Neumann, J . and O. Morgenstern (1953) Theory of Games and Economic Behavior, 3rd edition, Princeton: Princeton University Press.

Weber, R. (1994) "Games in Coalitional Form," in Handbook of Game The ory, Vol. II ed. by R. Aumann and S. Hart. Amsterdam: Elsevier Science 